53. Jahrestagung der DGNR fand erstmals in Frankfurt am Main im Kap Europa statt

\title{
Gelungene Premiere in Frankfurt
}

Mit über 1.100 Besucherinnen und Besuchern war die neurorad-Premiere 2018 ein voller Erfolg. Unter dem Motto neuroRAD goes international konnte der Kongress unter der Leitung von Prof. Martin Wiemann aus Aachen viele internationale Gäste anziehen, die vor allem von dem IntensivHands-On der German Stroke School profitierten. Impressionen einer auf ganzer Linie erfolgreichen 53. Jahrestagung der Deutschen Gesellschaft für Neuroradiologie.

\section{Preisträger DGNR 2018}

Preis des Vereins für Hirn-AneurysmaErkrankte (Vortrag): Dr. Ruben MühlBenninghaus, Universitätsklinikum des Saarlandes, Klinik für Diagnostische und Interventionelle Neuroradiologie, Homburg. Transient In-Stent-Stenosis - A Common Finding after Flow Diverter Implantation

Preis des Vereins für Hirn-Aneurysma-Erkrankte (Poster): Mariya Pravdivtseva, Universitätsklinikum Schleswig-Holstein Campus Kiel, Klinik für Radiologie und Neuroradiologie, Kiel. Patient specific models for flow investigations of aneurysms

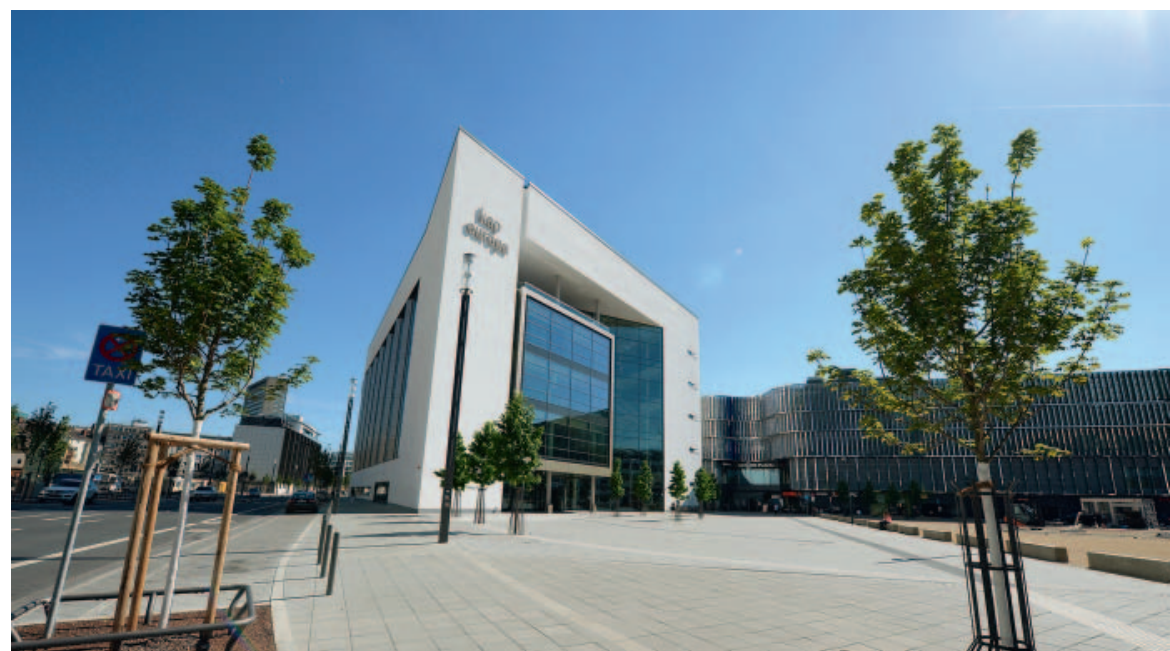

Austragungsort der DGNR Jahrestagung 2018 - 2020: Das Kap Europa im Frankfurter Messeviertel. @ Offenblende 2018.

1. Posterpreis: Dr. Annika Keulers, Uniklinik RWTH Aachen, Klinik für Diagnostische und Interventionelle Neuroradiologie, Aachen. Preventing vessel perforations in endovascular thrombectomy: Feasability and safety of passing the thrombus with a microcatheter without microwire: The wireless-microcatheter technique
2. Posterpreis: Prof. Dr. Omid Nikoubashman, Uniklinik RWTH Aachen Klinik für Diagnostische und Interventionelle Neuroradiologie, Aachen. Increased hemorrhage risk due to combined systemic thrombolysis and dual antiplatelet inhibition after endovascular stroke treatment 


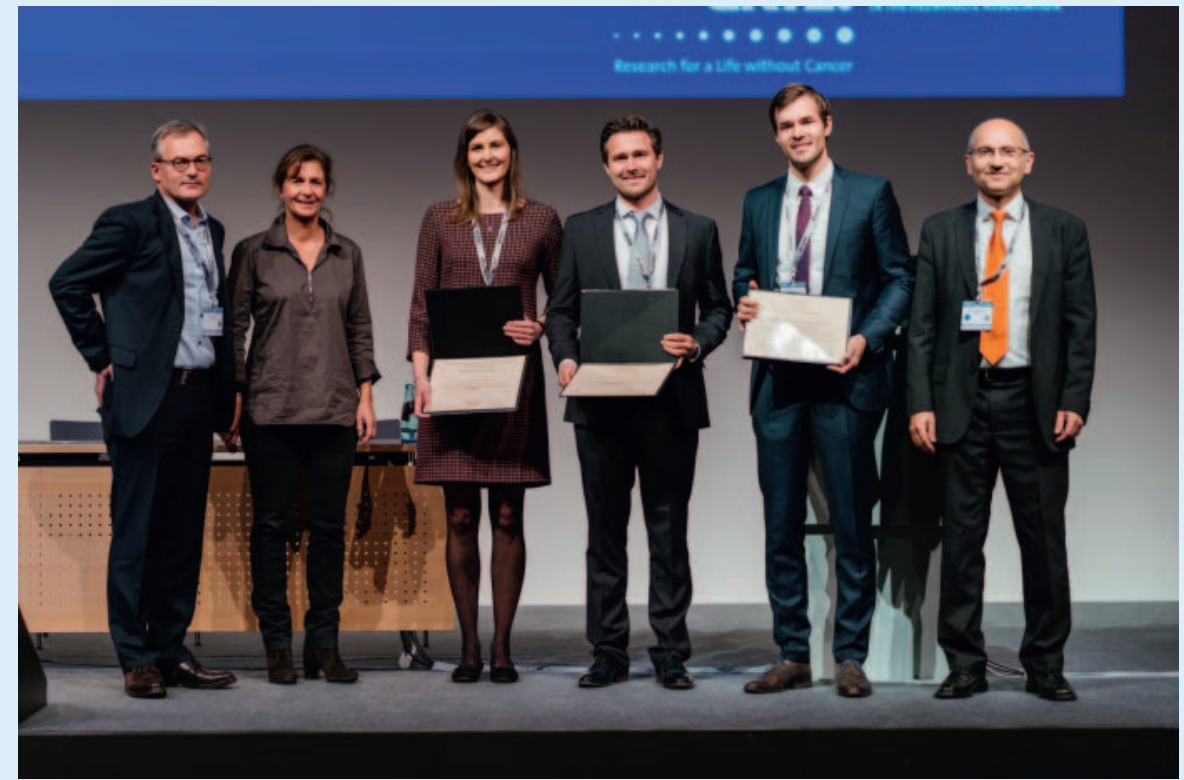

Gruppenbild der Preisträger 2018. Die Verleihung erfolgte in diesem Jahr am Kongressdonnerstag nach der Keynote-Session von Prof. Prayer und Prof. Rossi. @ O Offenblende 2018.

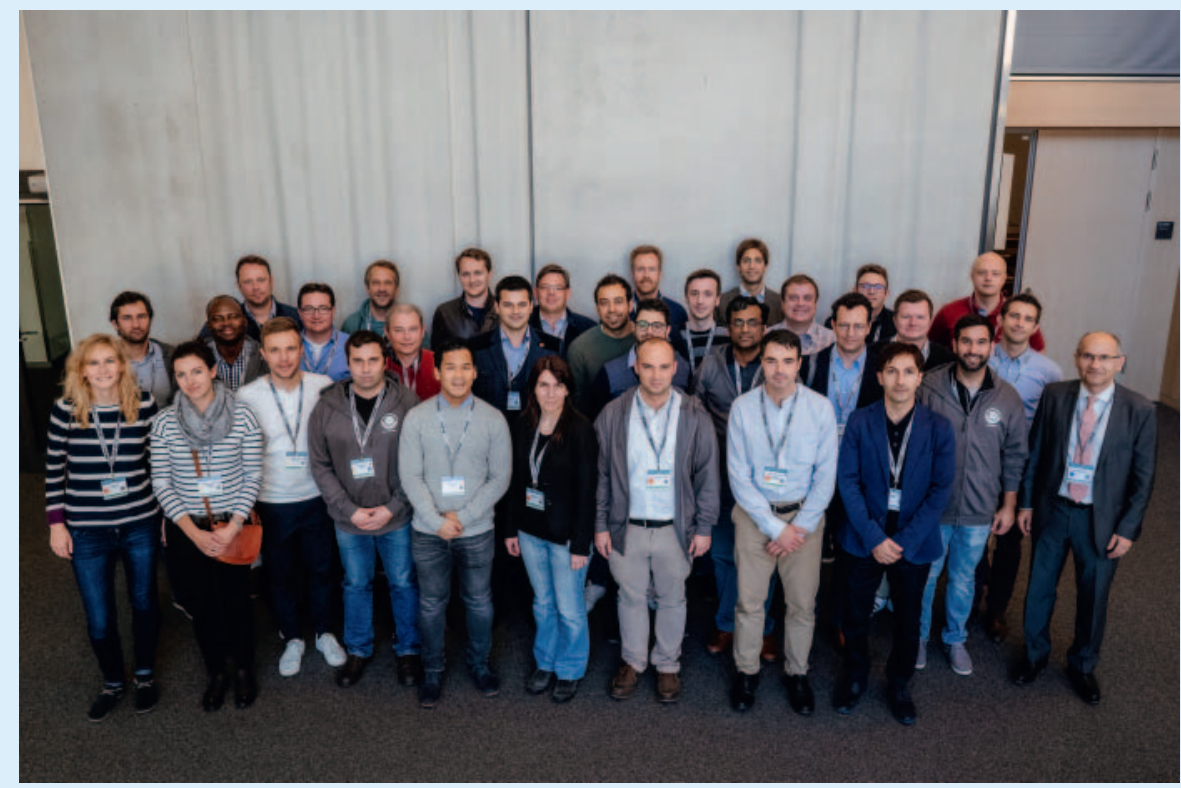

Gemeinschaftsgefühl: Drei Tage lang haben über 40 Kolleginnen und Kollegen aus den unterschiedlichsten Ländern die Mechanische Thrombektomie beim Schlaganfall erlernt Ganz rechts im Bild: Kongresspräsident und School-Verantwortlicher Prof. Martin Wiesmann. @ Offenblende 2018.

3. Posterpreis: Dr. Christian Maegerlein, Klinikum rechts der Isar der TU München, Abteilung für Diagnostische und Interventionelle Neuroradiologie, München. Pulling out all the stops to further reduce thrombus fragmentation during endovascular stroke treatment - the PROTECT PLUS technique
4. Posterpreis: Dr. Simon Schön, Klinikum rechts der Isar der TU München, Abteilung für Diagnostische und Interventionelle Neuroradiologie. Imaging glioma biology: spatial comparison of APT, CBV, DTI and FET

5. Posterpreis: Dr. Hani Ridwan, Uniklinik Aachen RWTH, Klinik für Diagnostische und Interventionelle Neuroradiologie,

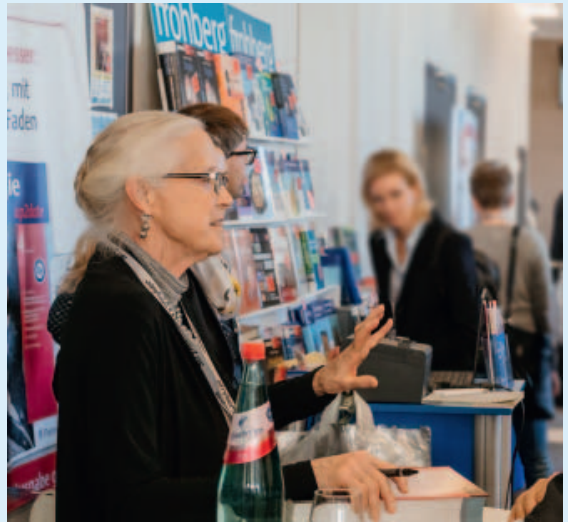

Der unbestrittene Star der 53. Jahrestagung: Prof. Anne Osborn (Salt Lake City), die mit ihrer Keynote Brain in Flame die Zuhörerschaft begeisterte. Hier beim signieren ihrer Werke. @ Offenblende 2018.

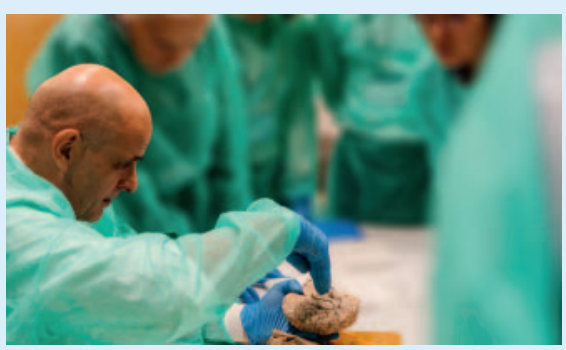

Eine der zahlreichen Neuerungen dieser Jahrestagung: Der Neuro-Anatomiekurs unter der Leitung des Leipziger Anatomen Prof. Ingo Bechmann. @ O Offenblende 2018.

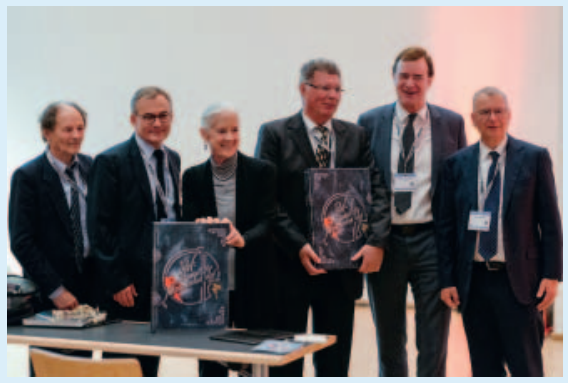

Die Ehrenmitglieder und ihre Laudatoren: Prof. Anne Osborn (Salt Lake City) und Prof. Friedhelm Zanella wurden zu Ehrenmitgliedern der Deutschen Gesellschaft für Neuroradiologie ernannt.@ Offenblende 2018.

Aachen. Endovascular Rescue Treatment for delayed cerebral ischemia following subarachnoid hemorrhage - A single Center analysis 


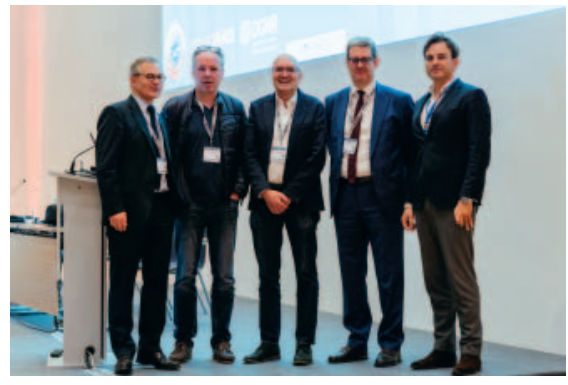

Alter und neuer Vorstand der DGNR: Mit dem Kongress begann die Amtszeit von Prof. Horst Urbach (Freiburg, in der Bildmitte), Prof. Michael Forsting (zweiter von links) und Prof. Jens Fiehler (erster von rechts) schieden aus dem Vorstand aus. (c) Offenblende 2018.
6. Posterpreis: Dr. Benedikt Wiestler, Klinikum rechts der Isar der TU München, Abteilung für Diagnostische und Interventionelle Neuroradiologie, München. Learning what's normal: Deep-learning human brain anatomy for unsupervised anomaly delineation in MR images

DGNR - Interventionspreis 2018: PD Dr. Wolfgang Kunz, LMU München, Klinik und Poliklinik für Radiologie, München. KostenEffektivität der Schlaganfall-Thrombektomie im Kontext klinischer Subgruppen, dem Patientenalter, der Zeit und der Reperfusion
Kurt-Decker-Preis 2018: Dr. Jennifer Kollmer, Universitätsklinikum Heidelberg, Neuroradiologie, Heidelberg. Charakterisierung diffuser Neuropathien mittels hochauflösender Magnet Resonanz Neurografie

Marc-Dünzl-Preis 2018: Dr. Daniel Paech, Deutsches Krebsforschungszentrum, Heidelberg. Chemical Exchange Saturation Transfer (CEST) Magnetresonanztomografie: Evaluation des diagnostischen Potentials bei Gliompatienten 